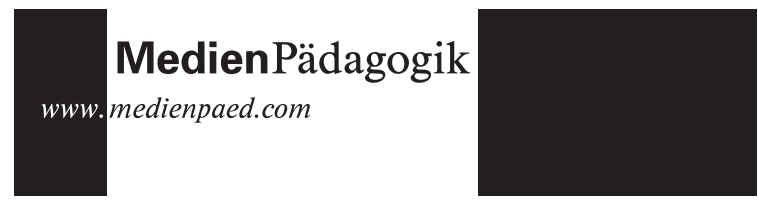

Andrea Pieter, Mandy Schiefner, Peter Strittmatter

17.9.2004

\section{Konzeption, Implementation und Evaluation von Online-Seminaren in der universitären, erziehungswissenschaftlichen Ausbildung}

Eine Vielzahl von Gründen sprechen für den Einsatz von Online-Seminaren in der universitären Lehre: Beispielsweise ermöglichen Online-Seminare eine Erweiterung des Veranstaltungsspektrums, eine Ausweitung der Wissensvermittlung sowie temporäre und örtliche Flexibilität (vgl. Scheuermann, 1998). Daneben sollte darauf geachtet werden, dass die Didaktik, die diesen Seminaren zu Grunde liegt, einen Schwerpunkt im Entwicklungsprozess bildet (vgl. Dörr \& Strittmatter, 2002). Im Folgenden werden exemplarisch zwei Seminare aus dem Hochschulbereich dargestellt, die diesen Anforderungen in ihrem konzeptionellen Aufbau gerecht werden wollen und hinsichtlich ihrer Akzeptanz überprüft wurden.

Die neuen Techniken sollen ein Mittel zur Reform darstellen. Sie sollen die Lehre verbessern, Themen neu aufbereiten und auffächern sowie die Studierenden animieren, Inhalte selbstständig zu erarbeiten und zu erforschen. Angesichts der ständig wachsenden Anforderungen an die Qualität der Hochschulausbildung und der auch künftig zunehmenden Kapazitäts- und Ressourcenprobleme an den deutschen Hochschulen müssen die neuen Informations- und Kommunikationstechnologien stärker als bisher genutzt werden. Mit diesen neuen Technologien ist es möglich, in Lehre und Studium eine neue Qualität zu erreichen. Beides kann zeitlich und räumlich vom bisherigen Semesterturnus entkoppelt und damit flexibler gestaltet werden. Der Studierende kann sein Studium besser seinem individuellen Lern- und Lebensweg anpassen. Das Studium schafft durch die Vernetzung individuelle Wahlmöglichkeiten, die bisher nicht so leicht umzusetzen waren.

Der Forschungsstand bezüglich Lernen mit (Neuen) Medien ist sehr differenziert zu betrachten. Etliche Ergebnisse aus Studien lassen sich nicht verallgemeinern, da sich diese auf vielerlei Einflussfaktoren (Art der Untersuchung, Stichprobe, ...) zurückführen lassen. Bezüglich der Qualitätsevaluation kann zudem kein einheitlicher Ansatz und keine einheitliche Funktion benannt werden, da sich die verschiedenen Qualitätsevaluationen erheblich untereinander unterscheiden.

Lernen mit neuen Medien ist nicht wie erwartet im Laufe der Zeit erfolgreicher geworden (Tergan, 2004). Dies liegt zum einen an unrealistischen Erwartungen, wie zum Beispiel verkürzte (Untersuchungs-) Statements wie «Interaktives Lernen fördert Wissenserwerb» und weiterhin an meist unangemessenen Untersuchungsmethoden (z. B. Medienvergleichsuntersuchungen). Aus diesem Grund sind die bisherigen Studien über Lernen mit Medien schwer zu beurteilen. Dies sind jedoch nicht die einzigen Fehleinschätzungen, die dem Lernen mit Neuen Medien zugrunde liegen.

Meist herrscht die implizite Annahme vor, dass eine optimale Gestaltung der Medien automatisch zu optimalen Lernprozessen und dies wiederum zu optimalen Lernerfolg führt. Dabei wird weder den Wechselwirkungen zwischen Lerner, Medium und Lernvoraussetzungen Beachtung geschenkt, noch wird die Passung von Medien, Inhalten und Methoden, die das Lernen ausmacht, berücksichtigt (Kozma, 1994).

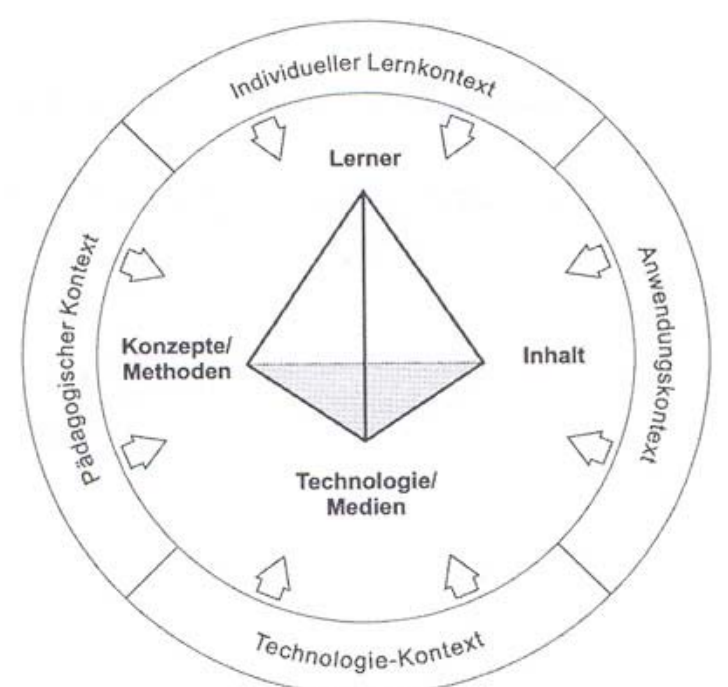

Abbildung 1: Lernrelevante Kontexte im E-Learning (Tergan, 2004) 
Deshalb werden in Zukunft verstärkt neue Elemente in die Evaluation von E-Learning eingebracht werden müssen, so zum Beispiel eine verstärkte Lernerorientierung, die Betrachtung von lernrelevanten Aktivitäten und Prozessen auf Seiten der Lernenden. Vor allem ist eine methodische Lücke zu schliessen, nämlich die Beurteilung der Wirkungen eines Lernangebotes (Tergan, 2004).

Die Grundvoraussetzung zur Implementation ist die Einbettung von ELearning in den Hochschulalltag und damit verbunden in das bestehende Curriculum (Seufert \& Euler, 2004).

In Bezug auf Nachhaltigkeit von E-Learning-Angeboten differenziert man Evaluationsstudien hinsichtlich «Lernumgebungen mit eMedien» und «Lernumgebungen mit eCommunication» (Seufert \& Euler, 2004).

Hinsichtlich dieser Unterscheidung kann man die nachfolgend vorgestellten Seminare eher der ersten Form zuordnen, denn bei dieser Form steht die multimediale Aufbereitung von Lerninhalten im Vordergrund. Allerdings sind auch Elemente der eCommunication durch den Tutor gegeben. In der Praxis sind die meisten eSeminarformen eher Mischformen.

Vor diesem Hintergrund wird in den folgenden Ausführungen die Implementation und Betreuung von Online-Seminaren im Fachbereich Erziehungswissenschaft vorgestellt (1), die didaktische Konzeption dieser Kurse dargestellt (2) und abschliessend das Ergebnis der ständig durchgeführten Evaluation diskutiert (3).

\section{Implementation und Betreuung der Seminare}

Bis dato wurden bereits drei Online-Seminare fertiggestellt, die thematisch dem Bereich «Lehren und Lernen» zuzuordnen sind. Die Veranstaltungen wurden in das Fakultätskonzept der Universität aufgenommen. Das heisst, die Seminare wurden in den Lehrveranstaltungskanon des Lehramtsstudienganges und des Magisterstudiengangs Erziehungswissenschaft der Universität des Saarlandes implementiert und werden dort semesterunabhängig angeboten. Ziel von Online-Seminaren in der erziehungswissenschaftlichen Ausbildung ist es, neben der Entwicklung und Erprobung von virtuellen Seminaren innerhalb des Grundlagenstudiums, den Studierenden während des Studiums einen angemessenen Kompetenzerwerb in Fragen der neuen Mediennutzung zu ermöglichen, in der Annahme, dass die Absolventen als Multiplikatoren beim Einsatz neuer Technologien im Unterricht fungieren können. Sie sollen die Lehre verbessern, Themen neu aufbereiten und auffächern sowie die Studierenden animieren, Inhalte selbständig zu erarbeiten und zu erforschen. Es wurde ein Lernserver aufgebaut, der stetig ausgebaut wird, indem OnlineVeranstaltungen geschaffen werden, die basierend auf geeigneten didaktischen Modellen und Instruktionsdesigns einerseits das selbstständige Lernen fördern (vgl. Strittmatter \& Niegemann, 2000) und andererseits auf viele, verschiedenste Lehrinhalte übertragbar sind (vgl. Bundesminsterium für Bildung und Forschung, 1999). Die Teilnahme an den Online-Seminaren ist für die Studierenden nicht verpflichtend, sondern als Zusatzangebot konzipiert. Jedoch haben sie mittlerweile einen festen Platz im Studium gefunden und werden gut frequentiert.

Die Seminare werden von Tutoren semesterunabhängig betreut. Die Nachfrage durch die Studierenden ist dabei in der vorlesungsfreien Zeit tendenziell höher als im laufenden Semesterbetrieb. Die Studierenden können sich einen individuellen Seminarablauf festlegen und u. a. auch selbst entscheiden, zu welchem Zeitpunkt das Seminar für sie mit dem Scheinerwerb abgeschlossen wird. Die Tutoren stehen den Lernern jederzeit im Lernprozess beratend zur Seite und sind via E-Mail oder Diskussionsforen zu erreichen. Neben der Beratung bei technischen Problemen, Systemvoraussetzungen, Organisation des Seminarablaufs etc. fällt ihnen hauptsächlich die Aufgabe zu, bei Problemen, die den Lerninhalt betreffen, den Lerner zu unterstützen. Dies zum einen bei Verständnisproblemen und auftretenden Fragen und zum anderen im Rahmen der von den Studierenden zu bearbeitenden Arbeitsaufträge in den Kursen. Sie geben jedem Lerner ein zeitnahes und transparentes Feedback bezüglich der eingereichten Ausführungen zu den jeweiligen Aufgabenstellungen (vgl. Kapitel 2). Die Kommunikation im Kurs läuft über E-Mail-Kontakt mit den Tutoren, die von jeder Stelle des Kurses aus zu erreichen sind und binnen 1-2 Tagen antworten oder im Rahmen von Diskussionsforen, die zu bestimmten Themengebieten angeboten werden und sowohl von den Tutoren als auch den übrigen Lernern frequentiert werden. Anfänglich wurden regelmässige Chats angeboten, diese wurden jedoch aufgrund der geringen Nachfrage von Seiten der Studierenden wieder eingestellt (vgl. Kapitel 3), genauso wie die anfängliche Präsenzsprechstunde, welche die Möglichkeit des Face-to-Face Kontaktes gewährleisten sollte. Auch sie wurde von den Studierenden nicht angenommen. Insgesamt hat sich im Laufe des Einsatzes der Kurse gezeigt, dass die an den Kursen teilnehmenden Lerner diese sehr gezielt besuchen. Der Kontakt beschränkt sich fast ausschliesslich auf den Austausch mit den Tutoren. Jedoch 
widersprechen die Angaben der Studierenden in der durchgeführten Evaluation (vgl. Kapitel 3) ihrem tatsächlichen Nutzungsverhalten. Daraus ergeben sich weitere Fragestellungen, deren Beantwortung eventuell eine Überarbeitung der Kursstruktur notwendig machen.

\section{Didaktische Konzeption}

Den zu Grunde liegenden Online-Seminaren ist gemein, dass sie als video-, audio- und textgestützte Lerneinheiten konzipiert sind. Die so vermittelten Kursinhalte werden noch durch eine Vielzahl von Zusatzmodulen erweitert. So stehen dem Lerner beispielsweise immer eine umfangreiche Hintergrundbibliothek, ein umfassendes Glossar, Zusammenfassungen der wichtigsten Kursinhalte und eine umfangreiche Mediathek, in welcher alle im Kurs befindlichen Ton- und Videodateien zum Download bereit stehen, zur Verfügung.

Die Studierenden müssen im Rahmen ihres Seminarbesuchs eine Reihe von Arbeitsaufträgen bearbeiten, die sie zum einen in Form einer Selbsttestung zum Zweck der reinen Selbstüberprüfung vornehmen können und zum anderen in Form von schriftlichen Stellungnahmen, die an die betreuenden Tutoren geschickt werden und zum Scheinerwerb erforderlich sind.

Die Selbsttestung umfasst pro Kurs eine Reihe von Fragen, wobei das Feedback durch das Programm automatisch generiert wird. Folgende Abbildung zeigt ein Beispiel für eine solche Selbsttestungsaufgabe:
Die Lehr-Lerntheorien von D.P. Ausubel und J.S. Bruner

Fachrichtung Erziehungswissenschaft - Lehrstuhl Prof. Dr. Strittmatter Vorhergehende Seite | Nächste Seite

Übungsaufgaben

1. Wie begriundet Ausubel seine Kritik an Bruners Theorie, dass entdeckendes Lernen nicht zwangsläufig Lernen
bedeutet?

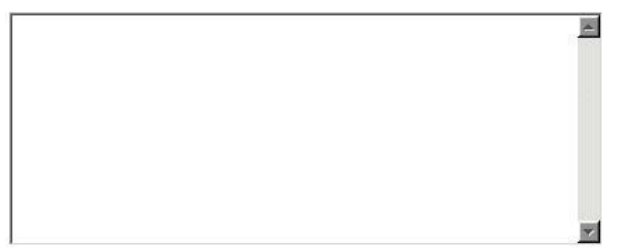

\begin{tabular}{l|l} 
Autgabe bestätigen & Korrekte Lösung?
\end{tabular}

2. Was schätzt Ausubel noch wichtiger ein als den Erwerb von Problemlösestrategien?

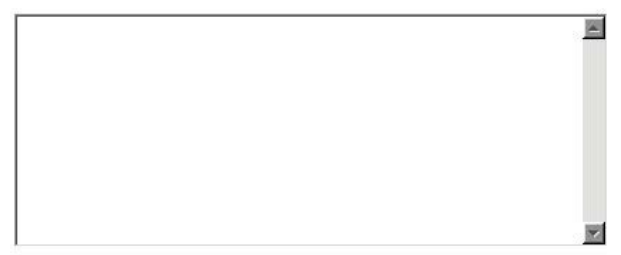

\begin{tabular}{l|l} 
Aufgabe bestätigen & Korrekte Lösung?
\end{tabular}

Abbildung 2: Beispiel für Selbsttestung im Rahmen des Kurses «Die LehrLerntheorien von D. P. Ausubel und J. S. Bruner

Nach der Beantwortung der Frage erhält der Lerner entweder eine aufmunternde Bestätigung, falls richtig geantwortet wurde, oder die korrekte Lösung mit Hinweisen, wo diese im Kurs oder in weiterführender Literatur zu finden ist. Das einfache Abrufen der Lösung ohne vorherige Antwort ist nicht möglich. Eine andere Art der Wissensüberprüfung liegt in Form von Arbeitsaufträgen in den Kursen. Dabei handelt es sich um breiter gefasste Fragestellungen, die einer umfangreicheren Rückmeldung des Lerners an die Tutoren bedürfen. Die Lerner erhalten von den Tutoren zeitnah ein aussagekräftiges Feedback (Abbildung 3 nimmt hierauf Bezug). 


\section{Arbeitsaufträge zu Modul 1}

1. Welche Ansatzpunkte (Möglichkeiten) zur Verbesserung der Schulleistung lassen sich ausmachen a) im eindimensionalen Modell

b) im mehrdimensionalen Modell ?

Führen Sie bitte jeweils möglichst viele Punkte auf!

2. Ein Schüler hat in einer Mathematikklassenarbeit 3 von 7 Aufgaben gelöst. Durchschnittlich wurden von den Schülern in dieser Klasse 3,14 Aufgaben richtig gelöst. Unterscheiden Sie bitte anhand dieses Beispiels:

- Leistungsfeststellung

- Leistungsbewertung

- Leistungsrückmeldung

Bewerten Sie die Leistung und formulieren Sie eine Rückmeldung. Welche Probleme entdecken Sie auf diesen drei Ebenen des Leistungsbewertungsprozesses (Beispiele: Deutschaufsatz, Mathematikarbeit)?

Abbildung 3: Beispiel für einen Arbeitsauftrag im Kurs «Leistungsbeurteilung und Lernmotivation»

Die Seminare stehen wahlweise als Online-Version oder CD-Rom-Version zur Verfügung. Jeweils die erste Lerneinheit der Kurse ist ohne Anmeldung frei zugänglich.

\section{Das Seminar Leistungsbeurteilung und Lernmotivation}

Das Seminar besteht aus insgesamt 10 Lerneinheiten, die sich mit den Themen: Zensuren und Zeugnisse, Klassenarbeiten, mündliche Prüfungen, Diagnosebogen, Schultests, Prozess der Urteilsbildung, Bezugsnormorientierung und subjektive Theorien von Lehrern befassen. Die einzelnen Seiten der Lerneinheiten sind nach folgendem Prinzip aufgebaut: Auf der linken Bildschirmhälfte finden die Lerner die im Video angesprochenen Lernmaterialien, Tafeltexte, Folien etc., die auch unter dem Button Bibliothek zum Download bereit stehen. Auf der rechten Bildschirmseite befinden sich die Videos, Literaturhinweise sowie interne und externe Links ins WWW, die thematisch eng mit dem Thema der jeweiligen Seite verbunden sind und ständig aktualisiert werden.
Die Klasse der halboffenen Aufgaben

(Zwischenformen) umfasst im Einzennen

folgende Möglichkeiten:

1. Freiantwortautgaben

Die Aufgaben enthalten eine (oder metrere) Fragen. Die Antwort(en)

darauf hat der Adressat zu formulteren.
Beispiel: "Emittein Sie das Potenfial des Punktes P gegenuber Masse.

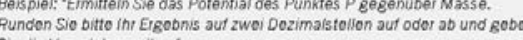

Sie die Vorzeichen mit an"

2. Assoziationsautgaben

Die Aufgaben enthatten Ausloser. Diesen Ausioser hat der Adressat
entsprechende andere, selbst zu formulierende, Elemente zu

assozileren
Beispiel: Nimm einen Allas und sien nach, an welchen Flussen oie

Beispiel: Nimm einen Aitos

Celle

Pusselsheim_ Heilbronn Wurzburg_

Abbildung 4: Ausschnitt einer Seite aus dem Kurs «Leistungsbeurteilung und Lernmotivation»

Die Videosequenzen auf den einzelnen Seiten haben eine maximale Länge von fünf Minuten, so dass die Online-Kosten bei akzeptabler Qualität des Videomaterials möglichst gering gehalten werden.

Die didaktische Grundlage dieses Seminars bilden das Instruktionsdesign von Gagné, Briggs \& Wagner (1987), das ARCS-Modell von Keller (1983, 1987) sowie die zehn Grundprinzipien nach Bourdeau \& Bates (1997). In diesen Theorien werden Lehrschritte postuliert, die im Rahmen des Seminars folgendermassen umgesetzt wurden (vgl. Pieter, 2002):

Einer jeden Lerneinheit sind sogenannte Advance Organizer vorgeschaltet, die über die Lernziele informieren bzw. vermitteln, welche Relevanz der Lernstoff im Seminarverlauf besitzt. Es handelt sich dabei um vorstrukturierende Lernhilfen, die dem Lerner verdeutlichen, welche Voraussetzungen er für das erfolgreiche Bearbeiten der Lerneinheit mitbringen sollte und was im Rahmen der Lerneinheit an Lernstoff auf ihn zukommen wird. 
Lerneinheit 1:

Organisation und Grundbegriffe

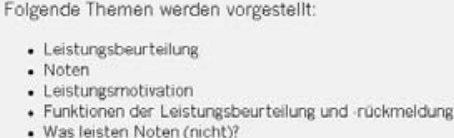

Abbildung 5: Beispiel für einen Advanced Organizer

Mittels Videosequenzen mit Ausschnitten aus einem Präsenzseminar zum gleichen Thema wird in dem Kurs die Aufmerksamkeit der Lerner gewonnen. Die Videosequenzen sind inhaltlich auf die Lernmaterialien angepasst. In einer empirischen Überprüfung des Videoeinsatzes konnte nachgewiesen werden, dass diese Einspielungen insgesamt zur Aufrechterhaltung der Motivation beitragen (vgl. Fey, 2002).

Der Lernstoff wird anhand charakteristischer Merkmale dargestellt. Beispielsweise erhalten die Lerner in einem Kapitel über die Bewertung mündlicher Prüfungen zwei Videoausschnitte von tatsächlichen mündlichen Prüfungen, die sie selbst bewerten sollen. Das Lernen wird zum einen durch Vorschläge zum Ablauf des Seminars angeleitet, kann aber von den Lernenden auch beliebig durchlaufen werden. Zu Beginn einer jeden Lerneinheit gibt es Informationen, welche Wissensgrundlagen zum erfolgreichen Bearbeiten notwendig sind. Im Rahmen von OnlineSelbsttestungen und Arbeitsaufträgen, die zur Korrektur an Tutoren geschickt werden, erhalten die Lerner in jeder Lerneinheit die Möglichkeit, ihr erworbenes Wissen anzuwenden und auszuführen. Die Aufgabenstellungen sind so gestaltet, dass sie das Behalten und den Transfer sichern. Nach dem Bearbeiten der Tests und Arbeitsaufträge erhalten die Lerner eine informative Rückmeldung bezüglich ihren Antworten. Die Rückmeldungen sind so angelegt, dass sie einerseits Wissensdefizite aufzeigen, andererseits bei erfolgreicher Bearbeitung eine motivierende Bestätigung im Sinne einer Kompetenzunterstützung und Förderung der Erfolgszuversichtlichkeit in Aussicht stellt.
Das Seminar Die Lehr-Lerntheorien von David P. Ausubel und Jerome S. Bruner

Bei der Umsetzung des Seminars zu den Lehr-Lerntheorien von David P. Ausubel und Jerome S. Bruner wurden eben diese beiden LehrLerntheorien als didaktische Grundlage verwandt. Das heisst, der Teil des Seminars, der sich mit den Grundlagen der Theorie Ausubels befasst, wird auch mittels der Grundlagen dieser Theorie (Ausubel, 1978) umgesetzt und analog dazu wird der Bruner-Teil des Seminars mittels der LehrLerntheorie von Bruner (1973) realisiert. Auch dieses Seminar ist modular aufgebaut. Der Ausubel-Teil wird gemäss dieser Theorie, in welcher der Lehrer als Wissensvermittler im Vordergrund steht, vom Lerner unter genauer Anleitung bearbeitet. Die einzelnen Lerneinheiten bauen inhaltlich aufeinander auf und müssen linear durchlaufen werden.

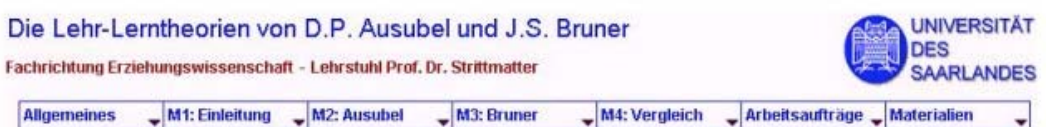

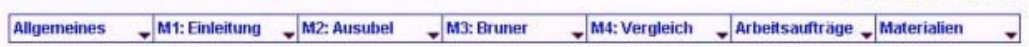

\section{Vorhergehende Seite | Nächste Seite}

Sie befinden sich in: Modul 2: Ausubel / Ubersich

\section{Modulübersicht}

Ausubels Theorie des expositorischen Lehrens

Folgende Themenschwerpunite werden in diesem Lernmodul behandelt

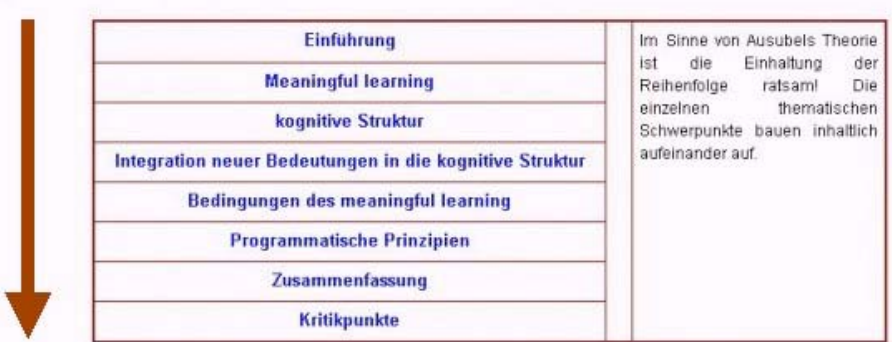

Vorhergehende Seite I Nachste Seite

Abbildung 6: Eingangsseite zum Teil der Ausubelschen Lerntheorien

Die Theorie Bruners, in deren Mittelpunkt das entdeckenlassende Lernen steht und dem Lehrer eher eine untergeordnete Rolle zukommt, kann selbstbestimmt bearbeitet werden, d. h. der Lerner kann frei wählen, in welcher Abfolge er die einzelnen Module der Theorie durchlaufen will. Die einzelnen Module bauen inhaltlich nicht aufeinander auf. 


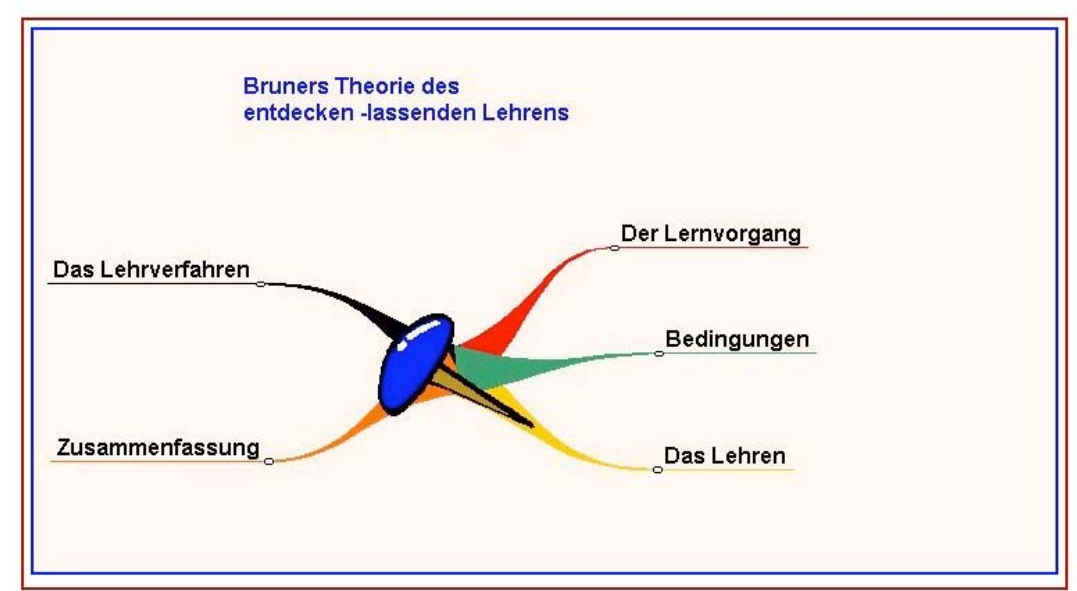

Sie haben nun die Möglichkeit dieses Modul ohne festgelegte Reihenfolge, gemäß der Theorie Eruners zu bearbeiten. Dazu können sie mit Hilfe der Übersicht navigieren. Die einzelnen Teile des Moduls sind voneinander unabhängig. Aber natürlich können Sie auch mit der Menüleiste am linken Bildschirmrand navigieren.

\section{Abbildung 7: Eingangsseite zum Teil der Brunerschen Lerntheorien}

Das Unterrichtsmaterial wird durch eine Vielzahl von Videoeinspielungen aus einem Lehrversuch einer Studentin ergänzt. Dies dient der Veranschaulichung der Umsetzungen der Lerntheorien im Unterricht. Darüber hinaus sind auf allen Seiten Tondateien eingefügt, so dass der Lerninhalt von den Lernern auch auditiv aufgenommen werden kann.

\section{Evaluationsergebnisse}

Die Qualität der angebotenen Kurse wird durch ständige interne und externe Evaluationen gesichert. Die externe Evaluation wurde vom Institut für Wirtschaftsinformatik der Universität St. Gallen durchgeführt. Im Folgenden werden die wichtigsten Ergebnisse des Evaluationsberichtes zum Seminar «Leistungsbeurteilung und Lernmotivation» dargestellt (Back \& Leithner, 2002). Der Bewertungsmassstab orientiert sich am Schulnotensystem in den Notenstufen sehr gut bis ungenügend. beziehen, während prozessorientierte Kriterien den Einsatz des Seminars und die Rahmenbedingungen bewerten.

\begin{tabular}{c|l} 
Produktorientierte Kriterien & Prozessorientierte Kriterien \\
\hline Motivation & Integration/Implementierung ins \\
Curriculum & Studium \\
Usability & Übertragbarkeit \\
Design & Qualitätssicherung
\end{tabular}

Die Aufrechterhaltung der Motivation im Verlauf des Seminars wurde mit gut bewertet. Gewährleistet wird dies u. a. durch überschaubare Lerneinheiten mit Angabe der Lernvoraussetzungen und der Lernziele, eine Lernkontrolle durch das Einsenden von Arbeitsaufträgen an einen Tutor sowie durch Videosequenzen und Online-Tests.

Das dem Kurs zu Grunde liegende Curriculum erhielt die Note sehr gut. Das Seminar ist in den Zyklus des Studiums der Erziehungswissenschaft eingebunden und ersetzt ein Präsenzseminar. Die Erlangung von Medienkompetenz auf Seiten der Studenten steht im Seminar im Mittelpunkt.

Das Kriterium der Usability wurde mit gut eingeschätzt. Die Benutzeroberfläche entspricht den Style Guides, die Navigation ist komfortabel. Als einziger Nachteil wurde die Nutzungsmodalität der Software Quick Time und die zum Zeitpunkt der Evaluation mässige Videoqualität angesehen. Doch dieser Aspekt wurde bereits verbessert; die Videos wurden überarbeitet und Quick Time wird nun direkt im Kurs zum Download angeboten.

Das Design wurde mit befriedigend bewertet. Bemängelt wurde, dass sich das Design an den Funktionen des Onlineseminars orientiert und der Drehort Hörsaal für eine unangenehme Atmosphäre sorgt.

Die Integration in das Studium ist nach dem St. Gallener Urteil sehr gut gelungen, wie dies auch von Seufert \& Euler (2004) gefordert wird. Dieses Seminar fällt unter die Pflichtveranstaltungen der Fachrichtung Erziehungswissenschaft und wird so gut in das Studium integriert. Ein Scheinerwerb ist semesterunabhängig möglich.

Die Rubrik Übertragbarkeit erhielt die Note befriedigend, da das Wissen stark an Mitarbeiter gebunden ist. Als positiv wurde gesehen, dass es einen Willen nach Kooperation mit anderen Hochschulen gibt und dass die 
Effizienz der Hochschullehre erhöht wird. Ein Relaunch des Kurses ist relativ einfach.

Die Qualitätssicherung wurde als befriedigend befunden. Derzeit findet eine ständige, formative Evaluation mittels Feedback-Fragebögen in den Online-Kursen statt.

Der didaktische Aspekt des Online-Seminars wird als gut angesehen. Es gibt zu jedem Thema eine Vorstellung der Lernziele, klare Formulierungen und Vorschläge bezüglich dem Lernvorgehen. Insgesamt bewertet das Institut für Wirtschaftsinformatik das Seminar mit der Note «gut» (2).

Daneben findet eine ständige interne Evaluation der Kurse statt. Im Folgenden werden die aktuellen Ergebnisse dargestellt.

An der Untersuchung nahmen insgesamt 23 Studierende teil, 7 Studierende besuchen den Kurs «Die Lehr- und Lerntheorien von Ausubel und Bruner», 15 den Kurs «Leistungsbeurteilung und Lernmotivation», eine Versuchsperson machte dazu keine Angabe. Vorab wurde das Nutzungsverhalten der Studierenden erfragt. Mit Abstand am häufigsten nutzen die Studierenden im Internet die Funktion E-Mail und das WWW.

Chats oder die Erstellung eigener Internetseiten werden von der Minderheit der Studierenden genutzt (73,9\% bzw. 69,6\% gaben an, diese Funktionen nicht zu nutzen). Newsgroups werden mit $56,6 \%$ häufiger besucht.

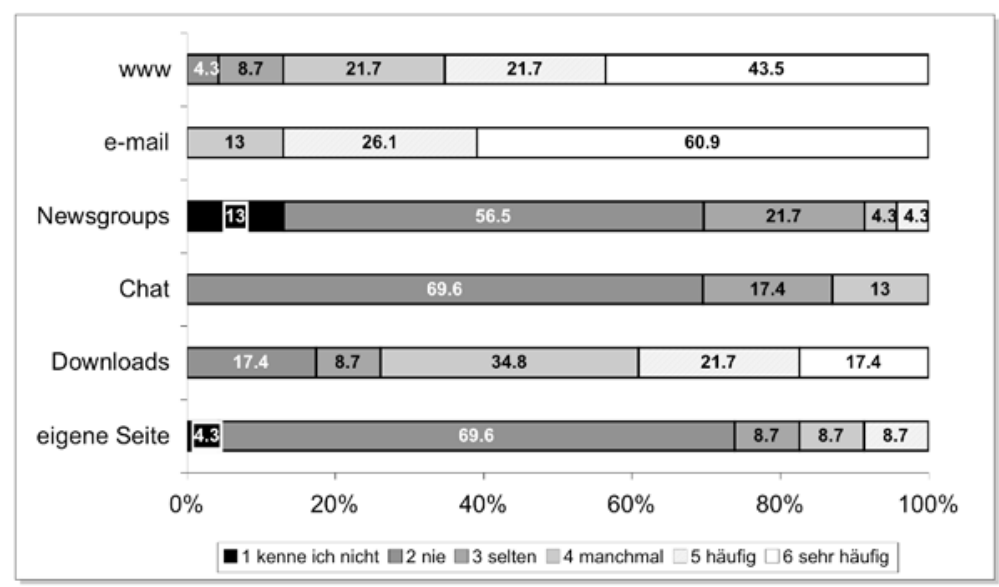

Abbildung 8: Nutzungshäufigkeit verschiedener Internetdienste

Ihr Interesse am Internet sowie ihre EDV-Kompetenz schätzen die Studierenden im Bereich gut bis sehr gut ein.

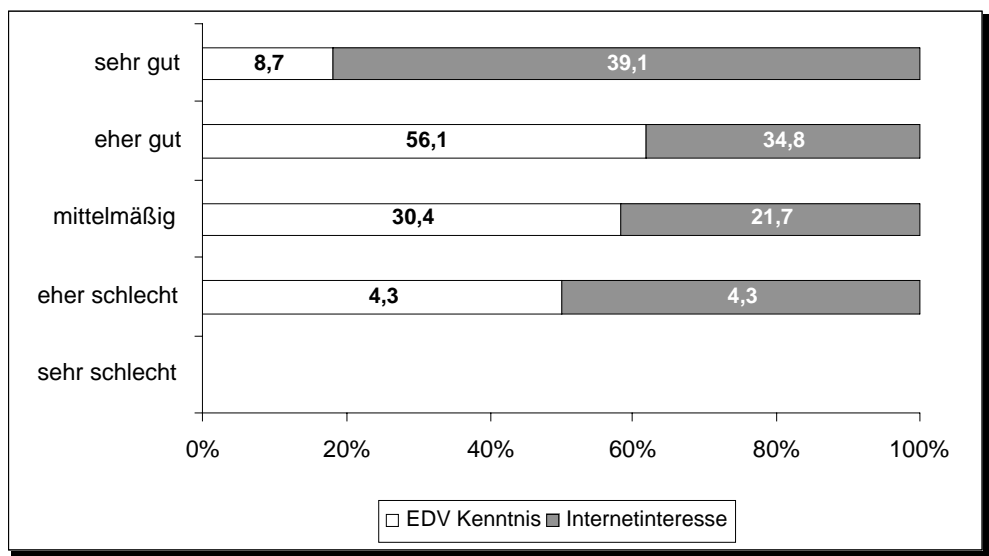

Abbildung 9: EDV Kenntnisse und Internetinteresse der Studierenden

Wichtig für die Bearbeitung eines virtuellen Seminars sind die Komponenten Optische Gestaltung und Einfachheit der Steuerung. Diese entscheiden darüber, wie sich Studierende in der Lernumgebung zurecht finden. Die folgende Abbildung zeigt die diesbezügliche Beurteilung der evaluierten Seminare:

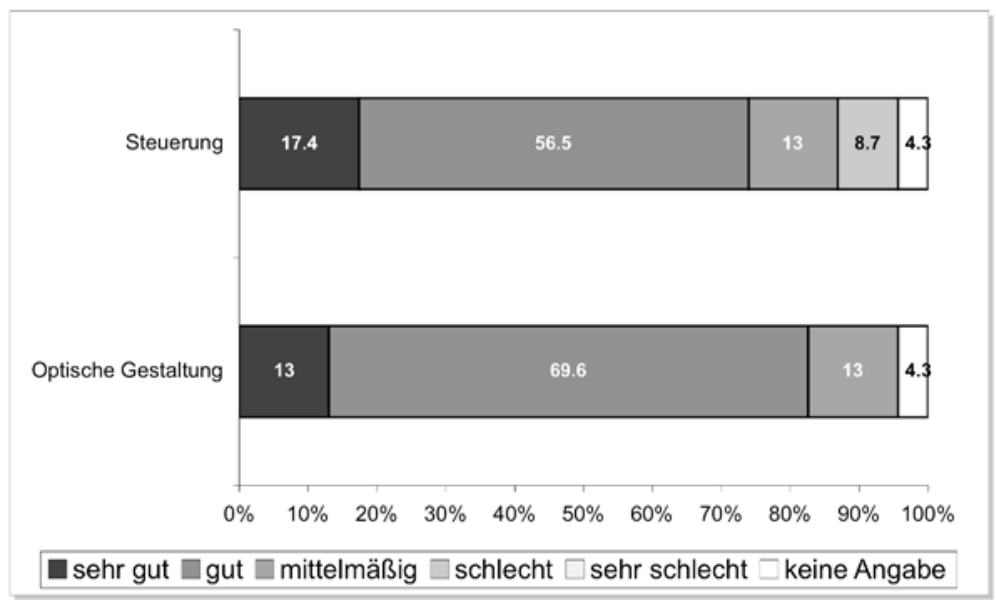

Abbildung 10: Einfachheit der Steuerung und Übersichtlichkeit der Optik 
Die Beurteilung einzelner Programmoptionen drückt in erster Linie die Qualität der technischen Umsetzung aus. Des Weiteren lassen sich jedoch auch qualitative Merkmale des Seminars anhand der Beurteilung von Programmoptionen aufzeigen.

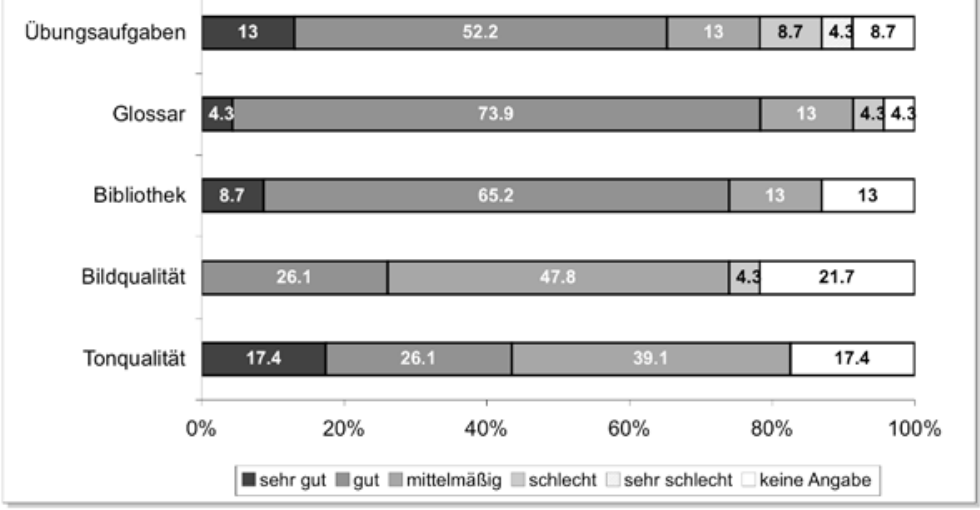

Abbildung 11: Einschätzung einzelner Komponenten des virtuellen Seminars

Es zeigte sich, dass vor allem das Glossar sowie die Bibliothek und die Übungsaufgaben von über zwei Drittel der Befragten als gut beurteilt wurde.

Im direkten Vergleich wurde auch nach der Wichtigkeit einzelner Programmaspekte gefragt. Dabei stellte sich heraus, dass die Studenten vor allem die Übungsaufgaben $(60,9 \%)$ als wichtig erachten, das Glossar wurde von $87 \%$ der Studenten als wichtig bis sehr wichtig angesehen, ebenso wie die Bibliothek (78,2\%). Die Bildqualität wird mit 21,7\% als sehr wichtig und mit $56,9 \%$ als wichtig eingeschätzt. Die Tonqualität übertrifft diese Werte noch: $26,1 \%$ finden die Tonqualität sehr wichtig, 60,9\% finden sie wichtig.

Die im Kurs verwandten Komponenten sind also Bestandteile, die in einem virtuellen Seminar nach Meinung der Studenten enthalten sein sollten.

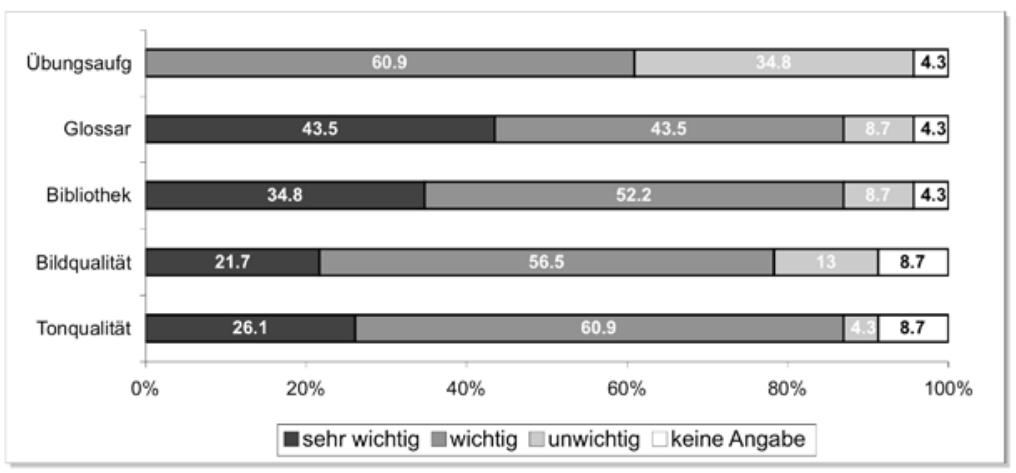

Abbildung 12: Wichtigkeit einzelner Programmaspekte

Um die Akzeptanz zum Thema Online-Lernen auf Seiten der Studierenden zu ermitteln, wurden sie auch gebeten, ihre Zustimmung bzw. Ablehnung zu vorgegebenen Statements zu geben. Für über die Hälfte der Befragten war die Rückmeldung durch die Tutoren schnell und passend, so dass es kaum zu Verzögerungen in ihrem Lernprozess kam. Der Arbeitsaufwand für die Bearbeitung eines Online Kurses wurde als vergleichbar mit dem Arbeitsaufwand eines Präsenzseminars angesehen. Für die Hälfte der Studenten ist in einem virtuellen Seminar der Austausch mit Kommilitonen wichtig. Obwohl die Studenten die angebotenen Sozialformen wie Präsenzsprechstunde oder das eingerichtete Forum kaum nutzen, gaben $69,6 \%$ der Studenten an, dass ihnen in einem virtuellen Seminar die Sozialkontakte fehlen. Dieses Ergebnis der Untersuchung macht eine Nachbefragung der Studenten nötig.

Von $86,9 \%$ der Studierenden wurde die mit dem Besuch eines OnlineSeminars verbundene Zeitersparnis als ein wichtiger Vorteil dieses Seminartyps angesehen. Gründe hierfür sind: Die Studierenden können das Seminar von zu Hause aus besuchen und müssen nicht extra zur Universität kommen. 


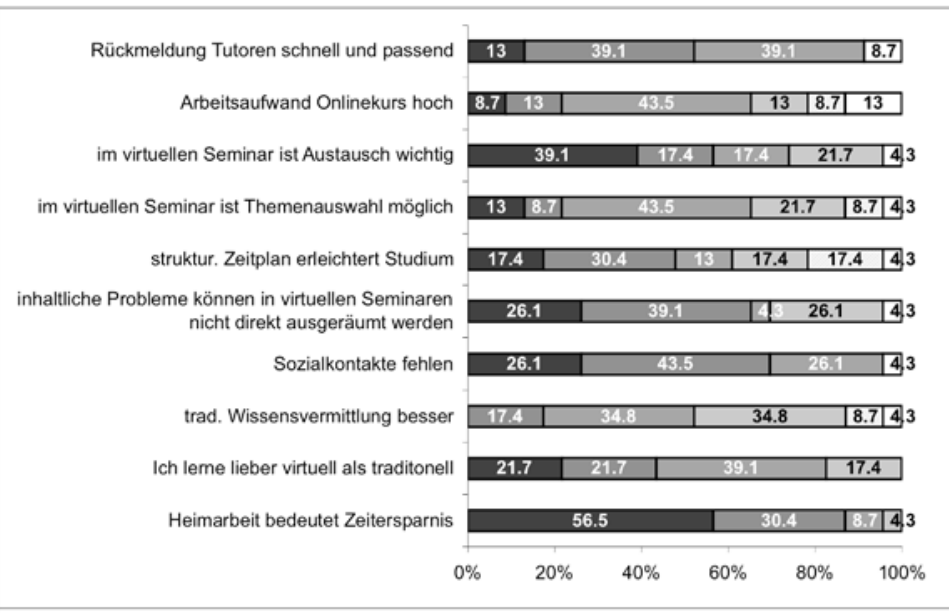

घstimme voll zu $\square$ stimme eher zu $\square$ weiß nicht $\square$ lehne eher ab $\square$ lehne völlig ab $\square$ keine Angabe

Abbildung 13: Zustimmung zu einzelnen Statements

Im letzten Teil der Evaluation konnten die Studierenden ihre direkte Meinung zu den Seminaren im Rahmen der Beantwortung offener Fragen abgeben.

Es stellte sich heraus, dass die Motivation zur Teilnahme an virtuellen Seminaren hauptsächlich in der besseren zeitlichen Einteilung für die Studierenden liegt. Die Studenten schätzen den Umstand, dass sie das Seminar selbst bestimmt von zu Hause aus bearbeiten können, als grossen Vorteil dieses Seminartyps ein. Des Weiteren gibt es die Möglichkeit, einzelne Themengebiete on demand nachzulesen. Aber nicht alle Themen lassen sich nach der Meinung der Studenten durch ein Online-Seminar bearbeiten; theoretische Grundlagenseminare eignen sich hierzu besser als Seminare mit eher praktischen Inhalten.

Die Frage nach dem persönlichen Benefit einer Teilnahme an OnlineSeminaren beantworteten viele Studenten in Hinblick auf die freie Zeiteinteilung, die es ermöglicht, dass Seminare durchaus auch in den Semesterferien ohne die Erhöhung der Semesterwochenstundenzahl zu absolvieren sind. Die eigene Leistung wurden besser eingeschätzt als in einem Präsenzseminar, weil ein selbstständiges Arbeiten gefördert wird. Bei einem Präsenzseminar würde man sich, nach Meinung der Studierenden, nur mit dem eigenen Thema in Referat und Hausarbeit auseinander- setzen, während man in einem Onlineseminar alle Themen bearbeiten muss und so einen besseren Überblick über das Seminar gewinnt.

Das Interesse für das Online-Lernen wurde bei vielen Studenten geweckt. Als Gründe hierfür gaben sie an, dass die Art eines Scheinerwerbs sehr angenehm sei, dass es eine sinnvolle Ergänzung der Lehre, vor allem für Studenten mit Kindern sei, dass es ein Lernen mit einer hohen Effektivität darstellt, dass Informationen vorhanden sind, die man immer wieder abrufen kann, dass es eine neue Form der Wissensvermittlung darstellt und eine einfache und problemlose Kommunikation erreicht wird.

Die Zeiten der Kursbearbeitung sind vielfältig. Es gibt praktisch keine Tages- oder Nachtzeit, die nicht als Arbeitszeit angegeben wurde. Hier sieht man in der Praxis ganz deutlich, dass Online-Lernen einen individuellen Lernprozess fördert, den jede Person zu jeder Zeit durchlaufen kann.

Es gab einige Elemente, die den Studenten besonders gut gefallen haben. Dies waren vor allem:

im Kurs «Leistungsbeurteilung und Lernmotivation» die Darstellung zweier verschiedener Prüfungen per Video

Aufbau und Struktur der Seminare

freie Einteilung des Klausurtermins

Navigation und Aufteilung des Kurses

sehr hilfsbereite und freundliche Mitarbeiter am Lehrstuhl

dass auch mal Studenten zu Wort kamen (in den Videos)

Das Vorlesen der Seiteninhalte in dem Kurs «Ausubel und Bruner» wurde von vielen Studenten als hilfreich angesehen, da es das Arbeiten auditiv unterstützt und eine Abwechslung beim Lernen und Lesen darstellt. Die Gefahr des Überlesens und des sturen Abarbeitens werde dadurch gemindert. Die Studenten, denen diese Feature nicht gefiel, gaben an, dass der Ton aber nicht störend sei, weil man ihn jederzeit abstellen könne.

Insgesamt sprechen die Evaluationsergebnisse dafür, dass es tatsächlich gelingt, im Studium eine neue Qualität durch Entkopplung des Semesterturnus und einer Flexibilisierung des Studiums zu erreichen. Die Studierenden passen die Seminare tatsächlich ihrem individuellen Lebensund Lernrhythmus an und schätzen die Möglichkeiten des selbstständigen Arbeitens, die diese Seminare bieten. Dafür, dass die Studierenden diese Zusatzangebote gerne annehmen, spricht auch das folgende Originalzitat aus der internen Evaluation: «Trotz der ganzen Kritik ein riesen Lob, dass 
es so ein Seminar überhaupt gibt. Die Sache ist absolut ausbaufähig und es lohnt sich bestimmt.»

\section{Fazit}

Zusammenfassend kann man konstatieren, dass sich der Aufbau von Online-Seminaren, die auf geeigneten didaktischen Modellen beruhen, im Rahmen der erziehungswissenschaftlichen Lehrerausbildung als auch im Magisterstudiengang Erziehungswissenschaft bis dato gelohnt hat und von den Studierenden sehr gut angenommen wird, wie die stetig ansteigende Zahl von Lernern zeigt. Darüber hinaus wird aufgezeigt, das in einigen Bereichen auch weiterhin noch eine Überarbeitung der Kurse durchgeführt werden muss (beispielsweise im Bereich der Kommunikation). Neben diesen Ergebnissen zur Akzeptanz sollte in zukünftigen Studien noch der Frage nachgegangen werden, ob die intendierten didaktischen Modelle auch tatsächlich für den Einsatz im Bereich des Online-Lernens geeignet sind und ob die damit implizierte Wirkung tatsächlich im Lernprozess der Studierenden zum Tragen kommt (angelehnt an Tergan, 2004). Die Evaluationsergebnisse zeigen auf, dass man auf dem richtigen Weg ist und die Studierenden das Online-Lernen als eine zusätzliche Lernform ansehen, die ihnen im Rahmen von Studienzeitverkürzung und einer zunehmenden Anzahl von Teilzeitstudierenden sehr entgegen kommt. Trotzdem wird es auch weiterhin nötig sein, Seminare dieser Art ständig zu evaluieren, zu überarbeiten und zu aktualisieren und letztlich immer wieder an die Lernervoraussetzungen anzupassen.

\section{Literatur}

Ausubel, D.P.; Novak, J.D. \& Hanesian, H. (1978). Educational Psychology. A Cognitive View (Second Editino). New York: Holt, Rinehart \& Winston.

Back, A. \& Leithner, B. (2002). Abschlussbericht des Doktorandenseminars Spezielles Thema I: Anwendungssysteme, Evaluation von ELearning Projekten. Unveröffentlichter Bericht. Universität St. Gallen.

Bourdeau, J. \& Bates, A. (1997). Instructional design for distance learning. In: S. Dijkstra, N.M. Seel, F. Schott \& R.D. Tennyson (Ed.). Instructional design. International perspectives. Mahwah, NJ: Erlbaum.

Bruner, J.S. (1973). Relevanz der Erziehung. Ravensburg: Otto Maier Verlag.

Bundesministerium für Bildung und Forschung (1999). Weiterbildungsinstitutionen, Medien und Lernumwelten. Bonn.

Dörr, G. \& Strittmatter, P. (2002). Multimedia aus pädagogischer Sicht. In: L.J. Issing \& P. Klimsa (Hrsg). Information und Lernen mit Multimedia und Internet. Lehrbuch für Studium und Praxis. 3. vollst. überarb. Auflage. Weinheim: Beltz.

Fey, A. (2002). Audi vs. Video : Hilft sehen beim Lernen? Unterrichtswissenschaft, 30, 4, S. 331-338.

Gagné, R.M., Briggs, L.J. \& Wager, W.W. (1988). Principles of instructional design. New York: Holt, Rinehart \& Winston.

Keller, J.M. \& Kopp, T.W. (1987). An Application of the ARCS model of motivational design. In: Reigeluth, Ch. (Hg.) Instructional theories in action. Lesson illustrating selected theories and models S. 289-320. Hillsdale, NJ: Erlbaum.

Keller, J.M. (1983). Motivational design of instruction. In C.M. Reigeluth (Ed.). Instructional design theories and models: an overview of their current studies. Hillsdale, NJ: Erlbaum.

Kozma, R. (1994). Will media influence learning? Reframing the debate. In: Educational Technology Research and Developement, 42, (2), S. 719.

Pieter, A. (2002). Universitäre Lehre online - ein Praxisbeispiel. Unterrichtswissenschaft, 30, 4, S. 304-314.

Scheeg, J. (2002). Projektevaluation «Virtuelles Lehren und Lernen im Hochschulbereich» am Beispiel des Kurses «Leistungsbeurteilung und Lernmotivation», Ausarbeitung im Rahmen des Doktorandenseminars «Spezielles Thema I: Anwendungssysteme» WS 2002/2003. 
MedienPädagogik

Scheuermann, F. (1998). Informations- und Kommunikationstechnologien in der Hochschullehre - Stand und Problematik des Einsatzes computerunterstützter Lernumgebungen. In F. Scheuermann; F. Schwab \& H. Augenstein (Hrsg.). Studieren und weiterbilden mit Multimedia. Perspektiven der Fernlehre in der wissenschaftlichen Aus- und Weiterbildung. Nürnberg: BW, Bildung und Wiss., Verlag und Software.

Seufert, S., Euler, D. (2004). Nachhaltigkeit von E-Learning-Innovationen. Ergebnisse einer Delphi-Studie. SCIL-Arbeitsbericht 2, SCIL,

University of St. Gallen, verfügbar unter:

http://www.scil.ch/publications/index-en.html

Strittmatter, P. \& Niegemann, H. (2000). Lehren und Lernen mit Medien. Darmstadt: Wissenschaftliche Buchgesellschaft.

Tergan, S.-O. (2004). Realistische Qualitätsevaluation von E-Learning. In: Meister, D.M.; Tergan, S.-O., Zentel, P. (Hrsg.). Evaluation von ELearning. Zielrichtungen, methodologische Aspekte, Zukunftsperspektiven. Münster: Verlag Waxmann, S. 131-154. 